\title{
Clozapine, Eating Disorders and Substance Abuse Obstacles to Innovation in a Peripheral Hospital
}

\author{
MICHAEL LAUNER
}

There appears to have developed in United Kingdom psychiatry a significant gap between academic teaching centres in some areas and the sharp end of service provision in the peripheral units. The innovator seems to be obstructed not only by this elitism but also by an overconcern for cheap simple care in the periphery which actively dissuades new developments.

Three fields are examined here in the same peripheral setting i.e. clozapine, eating disorder, and substance abuse, and each serves to demonstrate these worrying tendencies. This editorial explains how these obstructions have been overcome not only as a demonstration of technique but more importantly as a warning so that others recognise and do not have tc endure such short-sighted attacks on the proliferation of knowledge in the future.

Burnley is a declining industrial town in the northwest of England. In mental health the major development was the opening of one of the first psychiatric units in a district general hospital in the 1950s (Freeman \& Farndale, 1967). This rather revolutionary severing of links with the mental hospitals produced a few ripples but did cause silting up of beds with schizophrenic patients. This silting was not that great as many patients whose life quality was abysmal were cared for in a community with little expectation of anything different.

In the mid-80s the introduction of Community Psychiatric Nurses (CPNs) served merely to highlight the plight of chronic schizophrenics and increase the expectations of the community. In time the local CPNs drifted towards the 'worried well', possibly out of frustration with the lack of development of a psychiatric unit in a hospital whose priorities were dominated by the more acute specialities which generated more local support.

\section{Innovations}

\section{Clozapine}

In early 1990 clozapine was introduced to the UK and the Burnley unit began to use the drug, which requires regular blood monitoring, and set in motion the concept of clinic nurse and pharmacist combining to ensure patient compliance, with CPN backup for emergencies. Five patients were started on clozapine after the families had been educated about the sideeffects. The changes in these patients were noted to be quite different qualitatively from the changes due to conventional neuroleptics (Launer, 1991).

An early patient, a 40-year-old with a history of previous violence, began to become agitated about a girlfriend that he had known in his youth. He did not improve with psychotherapy and eventually returned to his psychosis, which did not respond to dose increase of clozapine. His mother demanded that we stop the 'experiment' and he was transferred back to high dose depot neuroleptics.

Some later patients experienced similar episodes and we felt that our observations needed to be disseminated. With links to academia being discouraged at the local centre of excellence for reasons of centralisation, research in the accepted sense was difficult. The hypothesis was that we were observing an awakening in patients after years of psychosis and if this was not recognised and dealt with appropriately, then not only treatment failures but also maybe tragedies may be expected. This was described in the only medium that was accessible to a peripheral unit - a national newspaper (the Daily Telegraph).

It was felt that if patients were at risk of awakening phenomena then the brunt of the burden was going to fall on the family. A family support group was initiated involving education and peer-support, and combinations of antidepressant medication and also oral conventional antipsychotic drugs were tried. The mother of the patient who had slipped back was persuaded to consent for her son to have clozapine again, this time in combination with conventional neuroleptics, and he was then able to be finally discharged.

The Americans rediscovered the awakening concept one year later and again chose a rather unconventional method of publication i.e. Time magazine, to international acclaim.

\section{Eating disorders}

In the early 1980s the services for eating disorders in the UK were scarce with the main units being based 
in London. The ability of a peripheral hospital to treat anorexics effectively was extremely limited and with the description of bulimia nervosa by Russell (1979) it became obvious that the Burnley service was going to be even less able to cope due to the complex nature of the syndrome.

Requests for investment in a service for eating disorders were declined in a district general hospital that did not even prioritise psychiatry. In 1983 the senior dietician, the only clinical psychologist and myself decided that we had to collaborate in order to offer an effective service, and without consultation we began an out-patient service which is now sited in a community health centre near to the hospital.

For clerical back-up a general practitioner clinical assistant session was traded in and in the first year we attracted around 80 referrals. The numbers are now near to 600 . We were concerned to provide some prescribed food supplements for the low weight anorexics and the more chaotic bulimics, to try and reduce the emotivity of food, and as there was none on the market we synthesised our own preparations. Eventually a letter to the $B M J$ (Launer, 1982) persuaded the Department of Health to allow some food supplements previously only used for metabolic disorders and malnutrition to be prescribed for eating disorders.

The absence of beds was highlighted in the Royal College of Psychiatrists report on eating disorders in 1989. At that time in the UK the provision of services was minimal and the number of beds worse at between 50-60, mainly in the southeast of England.

In addition the severity of the treatment regimes caused them not to be user friendly and was reflected in the self-help magazine 'Signpost' published by the Eating Disorder Association.

It was hypothesised that one of the core problems was due to low self-esteem. One patient had managed to return to normal weight by using the arts (stained glass) - this was again documented in a national newspaper. Some beds on a general psychiatric ward were vacated by the reduced need for hospitalising chronic schizophrenic patients due to the use of clozapine on a large scale, and a novel regime for the eating disorder patients was devised.

The food aspect of the therapy was transferred to the exclusive domain of the dietician, there was to be no reward and punishment system or target weights and the families of the patients were seen together for group therapy. The emphasis was on personal growth and the improvement of self-esteem. There was no set regime for all as each patient had her own programme. Some ideas for change within the patient were published locally in the form of a self-help handbook (Launer, 1994) and the concept of using creative work to stimulate the growth of self-esteem is encapsulated in a magazine inspired by a postulated 'language' used by eating disorder sufferers called 'Mespeak' described in the handbook. In addition a rating scale (not a subscale) for the measurement of self-esteem in eating disorders (Self-Esteem in Eating Disorders; SEED), is currently being standardised to enable measurements to be made of progress in treatment unrelated to weight and bingeing frequency.

\section{Substance abuse}

The abuse of illicit drugs in Burnley had been recognised since the 1960 s and the proliferation of drug-dealers and chaotic heroin addicts was felt to be a real threat to the community. In 1989 there was already needle exchange, methadone usage and a rudimentary community drugs team but it was difficult to distinguish dealers from sufferers and the side-effect of this problem was often violence. The GPs in the town were frightened of becoming involved and many addicts were not registered with the service or even a doctor.

The philosophy of methadone maintenance had become accepted nationally (HMSO, 1991) and what was needed was an effective system of control locally. The District recognised that there was a need and funding was made available for a pre-fabricated hut in the hospital grounds. The theory was that the new addicts were simply invited to attend and drink their methadone daily (level 1 ), with a view to being transferred to level 2 , i.e. a daily pick-up arrangement at selected retail pharmacists if they were suitable. The move on to level 3 was where GPs took over the care. Any transgressions and the offender went back to level 1 . This was simply a political solution to a clinical problem and its success can be measured by the tripling of the number of addicts who became registered, the lowering of the price of illicit opiates and a zero level of HIV in local addicts compared to up to $64 \%$ in Edinburgh and between $2-60 \%$ in Europe (HMSO, 1993).

\section{Conclusions}

The opposition to innovatory work in UK peripheral hospitals is both covert and direct. The lack of access to research manpower, funding and technical expertise is traditional in the periphery and the few clinicians that have triumphed, for example Steptoe and his test-tube babies and Charnley and his hip replacements, both in the northwest of England, have 
done so against the odds (Horrobin, 1990). Many established academic units are concerned with the growth of their own kudos both collectively and individually and there is little or no incentive to collaborate with the periphery.

The peripheral hospitals are tied to their commitment to 'bread and butter psychiatry' by short-sighted managers who are wary, sometimes to the extent of overt hostility, to innovations and research. The managers point out that any investment monies must be used for long-established and politically acceptable developments, hence the enthusiasm in Burnley for the treatment of substance abuse. More recently our earning power in the field of eating disorders has persuaded the local purchasers to buy a house near to the hospital for the care of six sufferers.

The only way that the less 'popular' subspecialities of intractable schizophrenia and eating disorder have been sustained has been through collective determination, single-mindedness and a hard-won financial argument about their earning and savings power in todays economically orientated internal market in the British health service.

If research and development is to continue to be funded externally as distinct from government aided grants, then centres must expect some form of quality control and they may have to seek to collaborate with proven external innovators to ensure survival.

One way to initiate these long overdue changes is to encourage innovators to take advantage of the facilities at the local centres of excellence and in return trainees could benefit from attachment to such peripheral centres and see a more realistic view of consumer-led research. Instead of the Burnley experience of a local centre of excellence rebuffing requests for links or even training posts and being forced to go to national units such as the Institute of Psychiatry for support, these centres must realise they can no longer rely on reputations sometimes established in the last century to retain crediblity in an increasing meritocratic world.

A credible starting point would be for some of the national bodies in the UK, such as the Medical Research Council or the Royal College of Psychiatrists, to invite some non-academics of proven merit to join their research committees.

\section{References}

Freeman, H. \& Farndale, J. (1967) New Aspects of the Mental Health Services. Oxford: Pergamon.

HMSO (1991) Drug misuse and Dependence, Guidelines on Clinical Management. Department of Health, Scottish Office Home and Health Dept. and Welsh Office.

(1993) AIDS and Drug Misuse Update, Report by the Advisory Council on the Misuse of Drugs. Department of Health.

HoRroBin, D. F. (1990) The philosophical basis of peer review and the suppression of innovation. Journal of the American Medical Association, 263, 1438-1441.

LAUNER, M. A. (1982) Problem awaiting solution. British Medical Journal, 285, 367.

(1991) Personal experience with clozapine. Psychiatric Bulletin, 15, 223-224.

(1994) Escape from the Ghetto of Despair. A Handbook for Sufferers from Eating Disorders. Burnley Health Care NHS Trust.

Royal College of Psychiatrists (1989) Report on Eating Disorders. London: RCPsych.

RusselL, G. (1979) Bulimia nervosa: An ominous variant of anorexia nervosa. Psychological Medicine, 9, 429-448.

Dr Michael Launer, MRCPsych, Burnley Health Care NHS Trust, Burnley General Hospital, Casterton Avenue, Burnley BB10 2PQ

(First received 19 December 1994, final revision 4 April 1995, accepted 19 May 1995) 